JOURNAL OF INTEGRAL EQUATIONS

AND APPLICATIONS

Volume 7, Number 1, Winter 1995

\title{
RUNGE-KUTTA TIME DISCRETIZATIONS OF PARABOLIC VOLTERRA INTEGRO-DIFFERENTIAL EQUATIONS
}

\author{
H. BRUNNER, J.-P. KAUTHEN AND A. OSTERMANN
}

\begin{abstract}
In this paper we prove optimal convergence results for implicit Runge-Kutta methods applied to parabolic Volterra integro-differential equations in Hilbert spaces. For this purpose, we show that such equations can be rewritten as parabolic differential equations in appropriate function spaces. Since Runge-Kutta methods are invariant under this transformation, it is sufficient to show that these methods converge for the resulting differential equations. While our theoretical framework is applicable to general (nonlinear) Volterra integro-differential equations, it is realized here in detail for the linear case.
\end{abstract}

1. Introduction. The aim of this paper is the study of the approximation properties of implicit Runge-Kutta methods for the abstract linear Volterra integro-differential equation (VIDE)

$$
y^{\prime}(t)+A(t) y(t)=f(t)+\int_{0}^{t} B(t, \tau) y(\tau) d \tau, \quad 0<t \leq T,
$$

subject to the initial condition $y(0)=y_{0}$ in a Hilbert space. We are mainly interested in the case where $A(t)$ and $B(t, \tau)$ are differential operators. More precise conditions will be given in Section 3 where we will specify the function spaces which form the framework of our analysis.

Abstract VIDEs of the general form (1.1) or of convolution type,

$$
y^{\prime}(t)+A y(t)=f(t)+\int_{0}^{t} B(t-\tau) y(\tau) d \tau
$$

Received by the editors on June 1, 1994, and in revised form on December 6, 1994.

Key words and phrases. Integro-differential equation, parabolic problem, RungeKutta method, fractional order of convergence.

1991 Mathematics Subject Classification. 65R20, 65J10, 45K05, 45L10.

Copyright (C1995 Rocky Mountain Mathematics Consortium 
have received considerable attention over the last 15 years. The recent monograph by Prüss [13] conveys a good picture of these developments; it also contains an extensive bibliography.

Much of this development has been motivated by partial VIDEs arising in the mathematical modeling of various physical phenomena, such as heat conduction in materials with memory (compare, e.g., Yanik and Fairweather [18] and Thomée [16] for relevant references on various applications of partial VIDEs). As a simple illustration, motivating the analysis presented in Section 3, we briefly recall a linear model for heat flow in a rigid heat conductor with fading memory [2]. It is given by the partial VIDE (the energy balance equation),

$$
\begin{aligned}
c_{0} & u_{t}(x, t)-\nabla \cdot\left(c_{1}(x) \nabla u(x, t)\right)+b(0) u(x, t) \\
& =r(x, t)-\int_{0}^{t}\left(\nabla \cdot(a(x, t-\tau) \nabla u(x, \tau))+b^{\prime}(t-\tau) u(x, \tau)\right) d \tau,
\end{aligned}
$$

with

$$
\begin{aligned}
u(x, 0) & =u_{0}(x), & x & \in \Omega \subset \mathbf{R}^{d} ; \\
u(x, t) & =0, & (x, t) & \in \partial \Omega \times(0, T] .
\end{aligned}
$$

Here $c_{0}>0$ and $c_{1}(x) \geq \gamma>0$ denote, respectively, the heat capacity and the thermal conductivity; $r(x, t)$ is the external heat supply. The unknown function $u(x, t)$ represents the temperature, and $a(x, t)$ and $b(t)$ are given (positive) memory functions.

Partial VIDEs like the one given above can be reformulated as abstract (parabolic) VIDEs (1.2) (or, in more general situations, (1.1)) in suitable function spaces.

The literature on the discretization of abstract VIDEs (1.1), (1.2), is now quite extensive; we refer the reader to the survey papers by Thomée [16] and Wahlbin [17] as well as to Tavernini [15], Sloan and Thomée [14], Yanik and Fairweather [18], Pani, Thomée and Wahlbin [12], Kauthen [9] and Zhang [19] for details and further references. Most of these papers consider only low-order time discretizations; moreover, the techniques employed in their analyses will not lead to optimal temporal convergence rates for higher-order methods.

It has recently been shown by Lubich and Ostermann [10] that strongly $A(\theta)$-stable Runge-Kutta methods for

$$
y^{\prime}+A(t) y=f, \quad y(0)=y_{0},
$$


exhibit a fractional order of convergence. More precisely, assume that $A$ is $\phi$-sectorial, with $\theta>\phi$. If the Runge-Kutta method has stage order $q$ and order $p \geq q+1+\beta$, then there result asymptotically sharp error estimates of the form $\mathcal{O}\left(h^{q+1+\beta}\right)$ where the value of $\beta \geq 0$ depends on the given (homogeneous or nonhomogeneous) Dirichlet or Neumann boundary conditions for the underlying partial differential equation. Similar results are valid for quasilinear and nonlinear parabolic equations, see $[\mathbf{1 0}]$.

The present paper applies this theory to abstract VIDEs

$$
y^{\prime}(t)=f\left(t, y(t), \int_{0}^{t} g(t, \tau, y(\tau)) d \tau\right) .
$$

We show that (1.5) may be written as a differential equation in a Hilbert space. This will be done formally in Section 2. In Section 3 we present the realization and the mathematical justification of these ideas for the VIDE (1.1). Although reminiscent of the direction chosen, e.g., in Grimmer [5], our approach uses a different framework; in particular, it is such that the transformation leading from (1.1) to the differential equation leaves the Runge-Kutta approximations invariant.

In Section 4 we establish the optimal (fractional) convergence rates indicated above, using the Runge-Kutta approximations to the differential equation. This section also includes a numerical illustration for these results. Finally, some additional remarks regarding possible extensions and applications may be found in Section 5 .

We conclude this section by introducing the Runge-Kutta terminology, see, e.g., Butcher [4] or Hairer and Wanner [7]. Consider the ordinary differential equation

$$
u^{\prime}=F(t, u), \quad u\left(t_{0}\right)=u_{0} .
$$

For a positive stepsize $h$ and $t_{n}=t_{0}+n h$, let $u_{n}$ denote the RungeKutta approximation of $u\left(t_{n}\right)$. This approximation is defined by the recursion

$$
\begin{aligned}
u_{n+1} & =u_{n}+h \sum_{j=1}^{s} b_{j} F\left(t_{n}+c_{j} h, U_{n j}\right), \quad n \geq 0, \\
U_{n i} & =u_{n}+h \sum_{j=1}^{s} a_{i j} F\left(t_{n}+c_{j} h, U_{n j}\right), \quad i=1, \ldots, s, n \geq 0 .
\end{aligned}
$$


This method has order $p$ if $\left\|u\left(t_{n}\right)-u_{n}\right\| \leq C h^{p}$ for $n h \leq$ const whenever $F$ is sufficiently smooth. It has stage order $q$ if, for all $i=1, \ldots, s$,

$$
\sum_{j=1}^{s} a_{i j} c_{j}^{k-1}=c_{i}^{k} / k, \quad k=1, \ldots, q .
$$

The stability function $R(z)$ of a Runge-Kutta method is defined by

$$
R(z)=1+z b^{\top}(I-z \mathcal{A})^{-1} \mathbf{1},
$$

where we used the notation

$$
\mathcal{A}=\left(a_{i j}\right)_{i, j=1, \ldots, s}, \quad b=\left(b_{1}, \ldots, b_{s}\right)^{\top}, \quad \mathbf{1}=(1, \ldots, 1)^{\top} .
$$

The Runge-Kutta method is $A(\theta)$-stable if $I-z \mathcal{A}$ is invertible in the sector $|\arg (-z)| \leq \theta$ and the stability function is bounded therein by 1. The method is strongly $A(\theta)$-stable if it is $A(\theta)$-stable, has invertible coefficient matrix $\mathcal{A}$ and if the value of the stability function at infinity, $R(\infty)=1-b^{\top} \mathcal{A}^{-1} \mathbf{1}$, satisfies $|R(\infty)|<1$.

2. Outline of the main ideas. We are interested in numerical methods for abstract Volterra integro-differential equations of the form

$$
y^{\prime}(t)=f\left(t, y(t), \int_{0}^{t} g(t, \tau, y(\tau)) d \tau\right), \quad y(0)=y_{0} .
$$

For this purpose we rewrite the integro-differential equation as an abstract differential equation. We then apply a Runge-Kutta method to this differential equation and obtain in this way a numerical method for (2.1). In this section we carry out this task heuristically. The exact formulation, including the definition of the appropriate function spaces, is given in the next section. There we illustrate the approach of this section for linear parabolic Volterra integro-differential equations.

To reformulate (2.1) as a differential equation, we first introduce a function $z(t, r)$ which, on the diagonal $r=t$, gives the lag term

$$
z(t, t)=\int_{0}^{t} g(t, \tau, y(\tau)) d \tau
$$


We define

$$
z(t, r)=\int_{0}^{t} g(r, \tau, y(\tau)) d \tau
$$

This approach has been pointed out to us by E. Hairer and Ch. Lubich [6]. It has the advantage that, in the context of parabolic integro-differential equations it leads to differential equations with sectorial operators. For Runge-Kutta methods applied to equations with sectorial operators, sharp error estimates are available [10]. We note that several other possibilities for defining $z$ can be found in the literature. We just mention Brunner and van der Houwen [3, p. 101] and Grimmer [5]. For our purposes, however, the latter approach does not yield the optimal orders of convergence.

We now consider the second variable of $z$ as a real parameter, differentiate (2.3) with respect to $t$ and obtain the differential equation

$$
\begin{aligned}
y^{\prime}(t) & =f(t, y(t), P(t) z(t, \cdot)), & y(0) & =y_{0}, \\
\partial_{1} z(t, \cdot) & =g(\cdot, t, y(t)), & z(0, \cdot) & =0 .
\end{aligned}
$$

Here $\partial_{1}$ denotes differentiation with respect to the first variable and the substitution operator $P(t)$ is defined for a continuous function $\psi$ by

$$
P(t) \psi=\psi(t) .
$$

We next apply a Runge-Kutta method to (2.4) which results in the following recursion (using $t_{n i}=t_{n}+c_{i} h$ ):

$$
\begin{gathered}
y_{n+1}=y_{n}+h \sum_{j=1}^{s} b_{j} f\left(t_{n j}, Y_{n j}, Z_{n j}\left(t_{n j}\right)\right), \\
z_{n+1}(\cdot)=z_{n}(\cdot)+h \sum_{j=1}^{s} b_{j} g\left(\cdot, t_{n j}, Y_{n j}\right), \\
Y_{n i}=y_{n}+h \sum_{j=1}^{s} a_{i j} f\left(t_{n j}, Y_{n j}, Z_{n j}\left(t_{n j}\right)\right), \\
i=1, \ldots, s,
\end{gathered}
$$




$$
\begin{gathered}
Z_{n i}(\cdot)=z_{n}(\cdot)+h \sum_{j=1}^{s} a_{i j} g\left(\cdot, t_{n j}, Y_{n j}\right) \\
i=1, \ldots, s
\end{gathered}
$$

Inserting recursively (2.7) into (2.9) and evaluating at $r=t_{n i}$ gives

$$
\begin{aligned}
Z_{n i}\left(t_{n i}\right)= & h \sum_{j=1}^{s} a_{i j} g\left(t_{n i}, t_{n j}, Y_{n j}\right) \\
& +h \sum_{\nu=0}^{n-1} \sum_{j=1}^{s} b_{j} g\left(t_{n i}, t_{\nu j}, Y_{\nu j}\right) .
\end{aligned}
$$

Here we used $z_{0}(\cdot)=0$. Inserting (2.10) back into (2.6) and (2.8) finally yields the following numerical scheme for equation (2.1):

$$
\begin{aligned}
y_{n+1}=y_{n}+h \sum_{j=1}^{s} b_{j} f\left(t_{n j}, Y_{n j},\right. & h \sum_{l=1}^{s} a_{j l} g\left(t_{n j}, t_{n l}, Y_{n l}\right) \\
& \left.+h \sum_{\nu=0}^{n-1} \sum_{l=1}^{s} b_{l} g\left(t_{n j}, t_{\nu l}, Y_{\nu l}\right)\right)
\end{aligned}
$$

$$
\begin{gathered}
Y_{n i}=y_{n}+h \sum_{j=1}^{s} a_{i j} f\left(t_{n j}, Y_{n j}, h \sum_{l=1}^{s} a_{j l} g\left(t_{n j}, t_{n l}, Y_{n l}\right)\right. \\
\left.+h \sum_{\nu=0}^{n-1} \sum_{l=1}^{s} b_{l} g\left(t_{n j}, t_{\nu l}, Y_{\nu l}\right)\right) \\
i=1, \ldots, s .
\end{gathered}
$$

We thus obtained an extended Pouzet-Volterra-Runge-Kutta method, see Brunner and van der Houwen [3].

The approach sketched above is, of course, not limited to RungeKutta methods. Any numerical method for differential equations can be used. The resulting methods for (2.1) all have in common that they treat the differential part and the lag term by the same numerical scheme. Since the differential equation (2.4) is partitioned 
in a natural way, different numerical methods for the two equations of (2.4) can be used (with possibly different stepsizes). Methods that treat the differential part and the lag term in a different way have been considered, e.g., by Sloan and Thomée [14]; see also Zhang [19].

In order to obtain convergence results for (2.11) and (2.12), it is sufficient to study the convergence of $(2.6)-(2.9)$. This will be done in Section 4 .

3. Abstract setting for linear parabolic problems. In the remainder of this paper, we illustrate the ideas developed in Section 2 for the linear parabolic Volterra integro-differential equation

$$
\begin{gathered}
y^{\prime}(t)+A(t) y(t)=f(t)+\int_{0}^{t} B(t, \tau) y(\tau) d \tau, \\
0<t \leq T, \quad y(0)=y_{0} .
\end{gathered}
$$

We are mainly interested in the case where $A$ and $B$ are differential operators. Therefore we study this equation in an abstract Hilbert space setting. As framework for the operator $A$, we use the setting of [11].

Let $V$ and $H$ denote two separable complex Hilbert spaces with norms $\|\cdot\|$ and $|\cdot|$, and scalar products $((\cdot, \cdot))$ and $(\cdot, \cdot)$, respectively. We assume that $V$ is continuously and densely embedded in $H$. This allows us to consider the evolution triple (see, e.g., Aubin [1, pp. 62-65])

$$
V \subset H=H^{\prime} \subset V^{\prime},
$$

where $V^{\prime}$ denotes the dual of $V$. Recall that this construction implies

$$
\left\langle v^{*}, v\right\rangle=\left(v^{*}, v\right), \quad \text { for all } v \in V, v^{*} \in H .
$$

Here $\langle\cdot, \cdot\rangle$ stands for the anti-duality between $V^{\prime}$ and $V$. The norm on $V^{\prime}$ will be denoted by $\|\cdot\|_{*}$.

We make the following assumptions on the data of (3.1), which must hold uniformly for all $t, \tau \in[0, T]$ :

$$
\begin{aligned}
& \text { the mappings } t \mapsto A(t) \in L\left(V, V^{\prime}\right) \text { and }(t, \tau) \mapsto B(t, \tau) \\
& \in L\left(V, V^{\prime}\right) \text { are continuous and possess sufficiently }
\end{aligned}
$$

many continuous derivatives; 


$$
\begin{aligned}
& \alpha+A(t): V \rightarrow V^{\prime} \text { has a uniformly bounded inverse } \\
& \text { for some } \alpha \geq 0
\end{aligned}
$$

$A(t)$ is sectorial on $V$ (cf., Henry [8, p. 18]), i.e., it is closed, has a dense domain and its resolvent satisfies

$$
\left\|(\lambda+A(t))^{-1}\right\|_{V \leftarrow V} \leq \frac{M_{A}}{|\lambda-\alpha|}
$$

in the sector $|\arg (\lambda-\alpha)| \leq \pi-\phi$ with $0<\phi<\pi / 2$;

$$
f:[0, T] \rightarrow V^{\prime} \text { is sufficiently smooth. }
$$

We now give the framework for the differential equation (2.4) which for (3.1) takes the form

$$
\Psi^{\prime}+K(t) \Psi=F, \quad \Psi(0)=\Psi_{0},
$$

where $\Psi(t)=(y(t), z(t, \cdot))^{\top}, \Psi_{0}=\left(y_{0}, 0\right)^{\top}, F(t)=(f(t), 0)^{\top}$, and

$$
K(t)=\left(\begin{array}{cc}
A(t) & -P(t) \\
-B(\cdot, t) & 0
\end{array}\right) .
$$

For (3.6) we consider the evolution triple

$$
V \times W \subset H \times W \subset V^{\prime} \times W
$$

where $W=H^{m}\left(0, T ; V^{\prime}\right)$, with the norm

$$
\|\psi\|_{W}=\sum_{k=0}^{m}\left(T^{2 k-1} \int_{0}^{T}\left\|\psi^{(k)}(r)\right\|_{*}^{2} d r\right)^{1 / 2} .
$$

We will always assume that $m \geq 2$ and note that the norm defined in (3.8) is equivalent to the standard norm on $W$. The operator $P(t)$ is a continuous projection from $W$ to $V^{\prime}$. It is defined in (2.5), and we have $\|P(t)\|_{V^{\prime} \leftarrow W}=1$.

To enter the Hilbert space setting of [10], it remains to show that the linear operator $K(t)$ has appropriate regularity properties. 
Lemma 1. Suppose that (3.2) and (3.4) hold. Then

a) the mapping $t \mapsto K(t) \in L\left(V \times W, V^{\prime} \times W\right)$ has $m-1$ bounded derivatives. For $m \geq 2$ it is, in particular, Lipschitz continuous.

b) $K(t)$ is sectorial on $V \times W$, i.e., it is closed and densely defined and its resolvent satisfies

$$
\left\|(\lambda+K(t))^{-1}\right\|_{V \times W \leftarrow V \times W} \leq \frac{M}{|\lambda-\kappa|}
$$

in the sector $|\arg (\lambda-\kappa)| \leq \pi-\phi$ with the same $\phi$ as in (3.4).

Proof. Part a) follows from (3.7) and the corresponding properties of $A(t)$ and $B(\cdot, t)$, see $(3.2)$. For $P(t)$ we have $\left\|P^{(k)}(t)\right\|_{V^{\prime} \leftarrow W}=T^{-k}$. This follows from (3.8). To prove part b), we note that the operator $K(t)$ is a bounded perturbation of the sectorial operator

$$
K_{0}(t)=\left(\begin{array}{cc}
A(t) & 0 \\
-B(\cdot, t) & 0
\end{array}\right)
$$

with domain $D_{V \times W}\left(K_{0}(t)\right) \supset D_{V}(A(t)) \times H^{m}(0, T ; V)$.

Example. Let $A$ and $B$ denote two second-order differential operators with smooth and bounded coefficients on a smooth and bounded domain $\Omega$. We assume that $A(t)$ is strongly elliptic, uniformly in $t$. We equip this operator with homogeneous Dirichlet boundary conditions. For the Sobolev spaces $V=H_{0}^{1}(\Omega), H=L^{2}(\Omega)$ and $V^{\prime}=H^{-1}(\Omega)$, the assumptions (3.2)-(3.4) are easily verified. In particular, equation (1.3), described in the introduction and written as an abstract integrodifferential equation, fits into this framework.

4. Convergence results. We now prove convergence of extended Pouzet-Volterra-Runge-Kutta methods applied to (3.1). These results rely on the reformulation of (3.1) as an abstract differential equation (3.6) and on convergence results for Runge-Kutta approximations for the latter, given in [10]. As the estimates stated below are in terms of the solution, we assume that the solution of (3.1) is sufficiently smooth in time. 
Theorem 1. For the numerical solution of (3.1)-(3.5) we consider a strongly $A(\theta)$-stable Runge-Kutta method (defined by (2.11) and (2.12)) with $\theta>\phi$, with stage order $q$ and order $p \geq q+1$. If $y^{(k)} \in L^{2}(0, T ; V)$ for $0 \leq k \leq q+1$ and $y^{(q+2)} \in L^{2}\left(0, T ; V^{\prime}\right)$, we have the error estimate

$$
\begin{aligned}
& h \sum_{n=1}^{N}\left\|y\left(t_{n}\right)-y_{n}\right\|^{2}+\max _{n=1, \ldots, N}\left|y\left(t_{n}\right)-y_{n}\right|^{2} \\
& \quad \leq C\left(h^{q+1}\right)^{2}\left(\sum_{k=0}^{q+1} \int_{0}^{T}\left\|y^{(k)}(t)\right\|^{2} d t+\int_{0}^{T}\left\|y^{(q+2)}(t)\right\|_{*}^{2} d t\right) .
\end{aligned}
$$

This bound is valid for $N h \leq T$ and the constant $C$ depends only on the Runge-Kutta method, on the constants in (3.2)-(3.4), and on T.

Proof. We use the notations introduced in Sections 2 and 3. In particular, $\Psi_{n}$ denotes the Runge-Kutta approximation of $\Psi\left(t_{n}\right)$. We have, for $r \in[0, T]$,

$$
\begin{aligned}
h \sum_{n=1}^{N} \| & y\left(t_{n}\right)-y_{n} \|^{2}+\max _{n=1, \ldots, N}\left|y\left(t_{n}\right)-y_{n}\right|^{2} \\
\leq & h \sum_{n=1}^{N}\left\|y\left(t_{n}\right)-y_{n}\right\|^{2}+h \sum_{n=1}^{N}\left\|z\left(t_{n}, r\right)-z_{n}(r)\right\|_{*}^{2} \\
& +\max _{n=1, \ldots, N}\left(\left|y\left(t_{n}\right)-y_{n}\right|^{2}+\left\|z\left(t_{n}, r\right)-z_{n}(r)\right\|_{*}^{2}\right) \\
\leq & h \sum_{n=1}^{N}\left\|\Psi\left(t_{n}\right)-\Psi_{n}\right\|_{V \times W}^{2}+\max _{n=1, \ldots, N}\left\|\Psi\left(t_{n}\right)-\Psi_{n}\right\|_{H \times W}^{2} .
\end{aligned}
$$

The last inequality follows from the embedding

$$
\left\|z\left(t_{n}, r\right)-z_{n}(r)\right\|_{*} \leq\left\|z\left(t_{n}, \cdot\right)-z_{n}(\cdot)\right\|_{W} .
$$

We now apply Theorem 3.2 of [10] to obtain the following bound for (4.2):

(4.3) $C\left(h^{q+1}\right)^{2}\left(\int_{0}^{T}\left\|\Psi^{(q+1)}(t)\right\|_{V \times W}^{2} d t+\int_{0}^{T}\left\|\Psi^{(q+2)}(t)\right\|_{V^{\prime} \times W}^{2} d t\right)$. 
Inserting the definition of $\Psi$ into (4.3) and using (3.2), we obtain the desired bound (4.1).

Remark. Theorem 1 remains valid for $p=q=1$ if $h^{q+1}$ is replaced by $h^{q}$ in (4.1). This includes the backward Euler method. Different convergence proofs for this scheme, combined with space discretization, can be found, e.g., in Sloan and Thomée [14] and Kauthen [9].

The convergence estimate in Theorem 1 is not sharp. In the following we will derive the optimal convergence rate. In addition to the conditions (3.2)-(3.5), we further require that

$$
\left\|B(r, t)(\alpha+A(t))^{-1}\right\|_{V \leftarrow V} \leq C
$$

and

$$
\left\|(\alpha+A(t))^{\beta}\left(\partial_{1}^{\mu} \partial_{2}^{k} B(r, t)\right)(\alpha+A(t))^{-\beta}\right\|_{V^{\prime} \leftarrow V} \leq C,
$$

for $0 \leq \mu \leq m$ and $0 \leq k \leq q$, uniformly in $r$ and $t$. The value of $\beta$ is specified in Theorem 2 below.

If the solution has more spatial regularity, we can now show the following improved temporal convergence rates.

Theorem 2. In addition to the conditions of Theorem 1 , let $p \geq q+2$. We further suppose that (4.4), (4.5) and the regularity assumptions $y^{(q+2)} \in L^{2}(0, T ; V)$ and $y^{(q+3)} \in L^{2}\left(0, T ; V^{\prime}\right)$ hold. Let $\beta \in[0,1]$ be such that $D_{V}\left((\alpha+A(t))^{\beta}\right)$ is independent of $t$ (with uniformly equivalent norms) and $(\alpha+A)^{\beta} y^{(k)} \in L^{2}(0, T ; V)$ for $0 \leq k \leq q+1$. Then we have the error estimate, valid for $N h \leq T$ :

$$
\begin{aligned}
& h \sum_{n=1}^{N}\left\|y\left(t_{n}\right)-y_{n}\right\|^{2}+\max _{n=1, \ldots, N}\left|y\left(t_{n}\right)-y_{n}\right|^{2} \\
& \leq C\left(h^{q+1+\beta}\right)^{2} \sum_{k=0}^{q+1} \int_{0}^{T}\left\|(\alpha+A)^{\beta} y^{(k)}(t)\right\|^{2} d t \\
& \quad+C\left(h^{q+2}\right)^{2}\left(\sum_{k=0}^{q+2} \int_{0}^{T}\left\|y^{(k)}(t)\right\|^{2} d t+\int_{0}^{T}\left\|y^{(q+3)}(t)\right\|_{*}^{2} d t\right) .
\end{aligned}
$$


The constant $C$ depends on the same quantities as in Theorem 1 as well as on the constants in (4.4) and (4.5).

Proof. The proof relies on the techniques used in the proof of Theorem 3.3 in $[\mathbf{1 0}]$. We have to estimate

$$
\sum_{n=0}^{N-1} \int_{t_{n}}^{t_{n+1}}\left\|(\varrho+K(\tau))^{\beta-1} K\left(t_{n}\right) \Psi^{(q+1)}(t)\right\|_{V \times W}^{2} d t
$$

for fixed $\tau$ and sufficiently large $\varrho \geq 0$. Using the representation $[8$, p. 25]

$$
(\varrho+K(\tau))^{\beta-1}=\frac{\sin (1-\beta) \pi}{\pi} \int_{0}^{\infty} \lambda^{\beta-1}(\lambda+\varrho+K(\tau))^{-1} d \lambda
$$

for $0<\beta<1$ and

$$
(\gamma+K(\tau))^{-1}=\left(\begin{array}{cc}
S(\tau) & \gamma^{-1} S(\tau) P(\tau) \\
\gamma^{-1} B(\cdot, \tau) S(\tau) & \gamma^{-1}+\gamma^{-2} B(\cdot, \tau) S(\tau) P(\tau)
\end{array}\right)
$$

with

$$
S(\tau)=(\gamma+A(\tau))^{-1}\left(I-\gamma^{-1} B(\tau, \tau)(\gamma+A(\tau))^{-1}\right)^{-1}
$$

it can be verified easily that the operator

$$
(\varrho+K(\tau))^{\beta-1} K\left(t_{n}\right)\left(\begin{array}{cc}
\left(\alpha+A\left(t_{n}\right)\right)^{-\beta} & 0 \\
0 & \left(\alpha+A\left(t_{n}\right)\right)^{-\beta}
\end{array}\right)
$$

is bounded on $V \times W$. This also holds for $\beta=0$ and $\beta=1$. Therefore, (4.7) can be estimated by a constant times

$$
\int_{0}^{T}\left\|(\alpha+A)^{\beta} y^{(q+1)}(t)\right\|^{2} d t+\int_{0}^{T}\left\|(\alpha+A)^{\beta} \partial_{t}^{q}(B(\cdot, t) y(t))\right\|_{W}^{2} d t .
$$

Due to (4.5) this is bounded itself by a constant times

$$
\sum_{k=0}^{q+1} \int_{0}^{T}\left\|(\alpha+A)^{\beta} y^{(k)}(t)\right\|^{2} d t
$$


The rest is now identical with the proof of Theorem 1 where $q$ is replaced by $q+1$.

Remarks. a) The above theorem yields the convergence rate $q+1+\beta$. In the example given at the end of the preceding section, $\beta$ is equal to $3 / 4-\varepsilon$ for arbitrarily small positive $\varepsilon$; see the example following Theorem 3.3 in [10]. Thus, for parabolic equations, Runge-Kutta approximations do not converge with their classical order $p$, but one observes an order reduction from $p$ to $q+1+\beta$, in general.

b) If (3.1) results from space discretization of a partial integrodifferential equation, with conditions (3.2)-(3.4), (4.4) and (4.5) holding uniformly in the meshwidth, then one has the estimate

$$
\begin{aligned}
h \sum_{n=1}^{N}\left\|\hat{y}\left(t_{n}\right)-y_{n}\right\|^{2}+\max _{n=1, \ldots, N}\left|\hat{y}\left(t_{n}\right)-y_{n}\right|^{2} \\
\quad \leq C\left(\mathcal{B}+\left|e_{0}\right|^{2}+|R(\infty)| h\left\|e_{0}\right\|^{2}+h \sum_{n=0}^{N} \sum_{j=1}^{s}\left\|d\left(t_{n j}\right)\right\|_{*}^{2}\right) .
\end{aligned}
$$

Here $\mathcal{B}$ denotes the righthand side expressions in (4.1) or (4.6), and $\hat{y}$ is a projection of the solution of the partial integro-differential equation onto the finite-dimensional approximation space, satisfying

$$
\begin{aligned}
\hat{y}^{\prime}(t)+A(t) \hat{y}(t) & =f(t)+d(t)+\int_{0}^{t} B(t, \tau) \hat{y}(\tau) d \tau, \\
\hat{y}(0) & =y_{0}+e_{0}
\end{aligned}
$$

with $d(t)$ denoting the space truncation error. This estimate can be proved as for parabolic differential equations; see the last remark in $[\mathbf{1 0}$, Section 3].

Numerical illustration. We solved the problem

$$
\begin{aligned}
u_{t}(x, t)-a(x, t) u_{x x}(x, t) & =f(x, t)+\int_{0}^{t} b(x, t, \tau) u_{x x}(t, \tau) d \tau \\
0 & \leq t \leq 1,
\end{aligned}
$$

with $u(x, 0)=u_{0}(x), x \in[0,1]$ and $u(0, t)=u(1, t)=0, t \in[0,1]$. 
Example 1. $u_{0}(x)=x(1-x), a(x, t)=1, b(x, t, \tau)=x(1+$ $t) \exp (-t \tau)$ and $f(x, t)$ such that the exact solution is $u(x, t)=x(1-$ $x) \exp (-t)$.

Example 2. $u_{0}(x)=x(x-1 / 3)(1-x), a(x, t)=2+\sin (x t)$, $b(x, t, \tau)=(1-2 x)(1+t) \exp (-t \tau)$ and $f(x, t)$ such that the exact solution is $u(x, t)=x(x-1 / 3)(1-x) \exp (-t)$.

We approximated the space derivatives by standard three-point finite differences; details for space discretization can be found in $[\mathbf{9}]$. For time discretization we used Radau IIA methods [7, p. 76] with $s$ stages $(s=1, \ldots, 5)$. These methods have order $p=2 s-1$ and stage order $q=s$. They are strongly $A(\theta)$-stable with $\theta=\pi / 2$ and satisfy $R(\infty)=0$. The temporal convergence rates we observed in the norm $\left(h \sum_{n=1}^{N}\left\|y\left(t_{n}\right)-y_{n}\right\|^{2}\right)^{1 / 2}$ are given in Tables 1 and 2 . They are in perfect agreement with the value of $\beta$ given above. One observes the order $\min \{p, q+1+3 / 4\}$.

TABLE 1. Convergence rates of $s$-stage Radau IIA methods for Example 1.

\begin{tabular}{|c|c|c|c|c|c|c|c|}
\hline$N$ & 2 & 4 & 8 & 16 & 32 & 64 & 128 \\
\hline$s=1$ & 1.04 & 1.04 & 1.04 & 1.02 & 1.01 & 1.01 & 1.00 \\
\hline$s=2$ & 2.54 & 3.11 & 3.02 & 2.96 & 2.95 & 2.96 & 2.97 \\
\hline$s=3$ & 2.42 & 3.97 & 4.35 & 4.54 & 4.65 & 4.69 & 4.72 \\
\hline$s=4$ & 5.70 & 5.91 & 5.84 & 5.86 & 5.79 & 5.78 & 5.78 \\
\hline$s=5$ & 7.39 & 7.07 & 6.90 & 6.81 & 6.79 & 6.79 & 6.81 \\
\hline
\end{tabular}

TABLE 2. Convergence rates of $s$-stage Radau IIA methods for Example 2.

\begin{tabular}{|c|c|c|c|c|c|c|c|}
\hline$N$ & 2 & 4 & 8 & 16 & 32 & 64 & 128 \\
\hline$s=1$ & 1.06 & 1.10 & 1.07 & 1.04 & 1.02 & 1.01 & 1.01 \\
\hline$s=2$ & 3.07 & 2.89 & 2.79 & 2.79 & 2.83 & 2.88 & 2.92 \\
\hline$s=3$ & 2.93 & 3.69 & 4.03 & 4.15 & 4.31 & 4.46 & 4.57 \\
\hline$s=4$ & 4.86 & 5.25 & 5.32 & 5.24 & 5.46 & 5.69 & 5.73 \\
\hline$s=5$ & 6.34 & 6.59 & 6.17 & 6.29 & 6.69 & 6.74 & 6.75 \\
\hline
\end{tabular}


We do not know if (4.6) is sharp in the norm $\max _{n=1, \ldots, N}\left|y\left(t_{n}\right)-y_{n}\right|$. We note, however, that we observed numerically the higher order $\min \{p, q+2+1 / 4\}$ for this norm.

5. Concluding remarks. We have chosen a Hilbert space setting for (3.1) because the convergence theory of Runge-Kutta methods for differential equations with time-dependent, unbounded operators in Banach spaces is not yet sufficiently developed. In the context of Banach spaces $X$ it would be natural to replace the space $H^{m}\left(0, T ; V^{\prime}\right)$ by $C^{m}(0, T ; X)$.

The same orders of convergence as in Theorems 1 and 2 remain valid for quasilinear integro-differential equations with possibly nonlinear kernels. For the corresponding framework for differential equations, we refer to $[\mathbf{1 0}]$.

We finally give a remark on the case where $f$ and $g$ of (2.1) take values in finite-dimensional spaces. Let $f:[0, T] \times \mathbf{R}^{d} \times \mathbf{R}^{l} \rightarrow \mathbf{R}^{d}$ and $g:[0, T] \times[0, T] \times \mathbf{R}^{d} \rightarrow \mathbf{R}^{l}$ be sufficiently smooth. Then (2.4) is an ordinary differential equation in $\mathbf{R}^{d} \times C^{m}\left(0, T ; \mathbf{R}^{l}\right)$ with bounded (nonlinear) operator. Due to its boundedness, no order reduction occurs and any numerical method for (2.4) converges with its (classical) order $p$. Therefore, the corresponding numerical scheme for (2.1) also converges with order $p$.

Acknowledgment. This research was supported by the Natural Sciences and Engineering Research Council of Canada (Research Grant OGP0009406). Part of this work was carried out during a recent visit of J.-P. Kauthen and A. Ostermann to the Department of Mathematics and Statistics at Memorial University of Newfoundland.

\section{REFERENCES}

1. J.-P. Aubin, Applied Functional Analysis, Wiley, New York, 1979.

2. A. Belleni-Morante, An integro-differential equation arising from the theory of heat conduction in rigid materials with memory, Boll. Un. Mat. Ital. B(5) 15 (1978), 470-482.

3. H. Brunner and P.J. van der Houwen, The Numerical Solution of Volterra Equations, North-Holland, Amsterdam, 1986. 
4. J.C. Butcher, The Numerical Analysis of Ordinary Differential Equations: Runge-Kutta and General Linear Methods, Wiley, Chichester, 1987.

5. R. Grimmer, Resolvent operators for integral equations in a Banach space, Trans. Amer. Math. Soc. 273 (1982), 333-349.

6. E. Hairer and Ch. Lubich, private communication.

7. E. Hairer and G. Wanner, Solving Ordinary Differential Equations II. Stiff and Differential-Algebraic Problems, Springer-Verlag, Berlin, 1991.

8. D. Henry, Geometric Theory of Semilinear Parabolic Problems, Lect. Notes Math. 840, Springer-Verlag, Berlin, 1981.

9. J.-P. Kauthen, The method of lines for parabolic partial integro-differential equations, J. Integral Equations Appl. 4 (1992), 69-81.

10. Ch. Lubich and A. Ostermann, Runge-Kutta approximation of quasilinear parabolic equations, Math. Comp., to appear.

11. - Linearly implicit time discretizations of nonlinear parabolic equations, IMA J. Numer. Anal., to appear.

12. A.K. Pani, V. Thomée and L.B. Wahlbin, Numerical methods for hyperbolic and parabolic integro-differential equations, J. Integral Equations Appl. 4 (1992), $533-583$.

13. J. Prüss, Evolutionary Integral Equations and Applications, Birkhäuser Verlag, Basel, 1993.

14. I.H. Sloan and V. Thomée, Time discretization of an integro-differential equation of parabolic type, SIAM J. Numer. Anal. 23 (1986), 1052-1061.

15. L. Tavernini, Finite difference approximations for a class of semilinear Volterra evolution problems, SIAM J. Numer. Anal. 14 (1977), 931-949.

16. V. Thomée, On the numerical solution of integro-differential equations of parabolic type, in Numerical Mathematics Singapore 1988 (R.P. Agarwal et al., eds.), Internat. Ser. Numer. Math. 86, Birkhäuser Verlag, Basel, 1988, 477-493.

17. L.B. Wahlbin, Finite element methods for evolution equations, in Advances in Numerical Analysis, Vol. I, (W. Light, ed.), Clarendon Press, Oxford, 1991, 1-33.

18. E.G. Yanik and G. Fairweather, Finite element methods for parabolic and hyperbolic partial integro-differential equations, Nonlinear Anal. 12 (1988), 785-809.

19. N.-Y. Zhang, On fully discrete Galerkin approximations for partial integrodifferential equations of parabolic type, Math. Comp. 60 (1993), 133-166.

Department of Mathematics and Statistics, Memorial University of Newfoundland, St. John's, Newfoundland, Canada A1C 5S7

Institut de Mathématiques, Université de Fribourg, CH-1700 Fribourg, SWIT ZERLAND

Institut für Mathematik und Geometrie, Universität Innsbruck, Technikerstrasse 13, A-6020 Innsbruck, Austria 